\title{
Designing Highly Structured Polycaprolactone Fibers using
}

\section{Microfluidics}

\author{
Farrokh Sharifi ${ }^{1}$, Diamant Kurteshi ${ }^{1}$, and Nastaran Hashemi ${ }^{1,2^{*}}$ \\ ${ }^{1}$ Department of Mechanical Engineering, Iowa State University, Ames, IA 50011, USA \\ ${ }^{2}$ Center for Advanced Host Defense Immunobiotics and Translational Comparative Medicine, Iowa \\ State University, Ames, IA 50011, USA \\ *nastaran@iastate.edu
}

\begin{abstract}
Microfibers are becoming increasingly important for biomedical applications such as regenerative medicine and tissue engineering. We have used a microfluidic approach to create polycaprolactone (PCL) microfibers in a controlled manner. Through the variations of the sheath fluid flow rate and PCL concentration in the core solution, the morphology of the microfibers and their cross sections can be tuned. The microfibers were made using PCL concentrations of $2 \%, 5 \%$, and $8 \%$ in the core fluid with a wide range of sheath-to-core flow rate ratios from 120:5 $\mu \mathrm{L} / \mathrm{min}$ to $10: 5 \mu \mathrm{L} / \mathrm{min}$, respectively. The results revealed that the mechanical properties of the PCL microfibers made using microfluidic approach were significantly improved compared to the PCL microfibers made by other fiber fabrication methods. Additionally, it was demonstrated that by decreasing the flow rate ratio and increasing the PCL concentration, the size of the microfiber could be increased. Varying the sheath-to-core flow rate ratios from $40: 5$ to $10: 5$, the tensile stress at break, the tensile strain at
\end{abstract}


break, and the Young's modulus were enhanced from 24.51 MPa to $77.07 \mathrm{MPa}, 567 \%$ to $1420 \%$, and 247.25 $\mathrm{MPa}$ to 539.70 $\mathrm{MPa}$, respectively. The porosity and roughness of microfiber decreased when the PCL concentration increased from $2 \%$ to $8 \%$, whereas changing the flow rate ratio did not have considerable impact on the microfiber roughness.

Keywords: Polymer Microfibers, Microfluidic Approach, Hydrodynamic Focusing, Polycaprolactone

\section{Introduction}

Fiber systems are becoming increasingly important for numerous biological applications, such as tissue engineering, as the fibers are able to guide cell growth, alignment, and migration (Chung et al., 2012; Hwang et al., 2008a). Additionally, the design of microfibers gives them the correct properties in order to perform drug delivery and drug release in the human body for medical purposes (Caplin et al., 2015; Tiwari et al., 2010). The fibers have high surface area-to-volume and strength-to-weight ratios. Some of them are permeable and can be woven into textiles (Boyd et al., 2013b). These properties allow microfibers to carry even delicate materials, such as water-soluble drugs, throughout a biological medium with good accuracy (Kraitzer et al., 2008; Saraf et al., 2010). This makes for safe insertion and transmittance of material used for treatment, demonstrating the effectiveness of microfibers in medicine. The method of generation of the microfibers plays a role in determining its viability in these types of applications.

Several approaches exist for the fabrication of microfibers from naturally derived or synthetic materials such as electrospinning, wetspinning, biospining, meltspinning, and the microfluidic techniques (Tamayol et al., 2013). Electrospinning is relatively a simple method and it is feasible to efficiently scale-up and control the involved parameters such as flow rate and voltage. However, 
there are some difficulties in the fabrication of thick, complex 3D scaffolds with this method (Deng et al., 2012; Hwang et al., 2008a). Additionally, electrospun microfibers are generally not easy to align and it requires extra care to ensure that the fibers are accurately aligned, especially because the randomly aligned fibers are not desirable for applications like growing nerve cells (Jung et al., 2009). Wetspinning is an efficient method for fabricating fibers with a wide range of diameters by changing the needle(s) diameter. Nevertheless, long exposure to chemicals during the fabrication process is required, which can be harmful to cells (Enea et al., 2011). Biospinning method is the process of fabricating silk fibers by insects. Silk has high tensile strength and is biodegradable. In addition, after chemical processing, it is non-cytotoxic and non-inflammatory. The major challenges of using biospun fibers are the limitation of resources, which makes it difficult for the scale-up process, as well as the fact that the process of silk fiber fabrication is time consuming (Reddy and Yang, 2010). In the meltspinning approach, various synthetic polymers can be used for fiber fabrication with this method. Fibers created by meltspinning have high mechanical properties. However, the meltspinning process is in a high temperature range $\left(150-300^{\circ} \mathrm{C}\right)$ and requires using expensive equipment. Using high temperatures during the fiber fabrication process prevents the cell or protein from being loaded onto the fiber in order to deliver the bioactive molecules in biomedical applications (Ella et al., 2011). Additionally, because the viscosity of the melted polymer is relatively high, a high pressure difference is needed to move the melted polymer through the spinneret (Akbari et al., 2011; Yim et al., 2006).

Using microfluidics to fabricate fiber is a relatively new approach in which the fiber is created in a microchannel using coaxial flow of core (pre-polymer) and sheath fluids. The key benefits of using this method include versatility of size, continuity of the fiber fabrication process, and simplicity of cell, protein or drug incorporation. This process is straightforward, cost-efficient, reproducible, and suitable for many biological applications since the fiber is created without using 
high temperature, high pressure, high voltages, or toxic materials. By changing the flow rate and flow rate ratio, the fiber size and aspect ratio can be simply controlled (Bai et al., 2014; Daniele et al., 2013; Goodrich et al., 2015; Hwang et al., 2008a). The microfluidic fiber fabrication can be employed to create fibers with various materials using different cross-linking mechanisms such as photopolymerization (e.g., polyethyleneglycol diacrylate, 4-hydroxybutyl acrylate) (Daniele et al., 2014a; Daniele et al., 2014b; Jeong et al., 2004; Oh et al., 2006), ionic gelation (e.g., alginate) (Shin et al., 2007), and thermal phase transition (e.g., agar) (Khademhosseini et al., 2006; VunjakNovakovic et al., 2004). However, there are some studies which employ phase inversion process instead of cross linking method to solidify the polymer (Bai et al., 2014; Hwang et al., 2008a). Hwang et al. used the solution of poly(lactic-co-glycolic acid) (PLGA) in dimethyl sulfoxide (DMSO) and mixture of glycerin and distilled water as the core and sheath fluids, respectively (Hwang et al., 2008a). At the fluid-fluid interface in the channel, the DMSO in the core fluid is replaced by water in the sheath fluid and the polymer is solidified. Likewise, Bai et al. dissolved gelatin in DMSO and showed that by exchanging the DMSO in the core fluid and ethanol in the sheath fluid, the gelatin can be solidified (Bai et al., 2014).

This approach makes it feasible to fabricate fibers with different shapes of solid (Bai et al., 2014; Daniele et al., 2013; Hasani-Sadrabadi et al., 2013), tubular (Choi et al., 2011; Kang et al., 2011), hybrid (Jung et al., 2009), and flat (Boyd et al., 2013a; Cho et al., 2012) dimensions for divergent applications such as cell encapsulation, alignment, and immobilization. There are different physical and chemical methods for solidification of fibers including diffusion-limited solidification by solvent extraction, diffusion-limited solidification by chemical cross-linking, and photo polymerization (Bai et al., 2014; Choi et al., 2011; Daniele et al., 2015; Yoo et al., 2015; Yoo et al., 2012). The fibers fabricated by photopolymerization are not easily degraded and metabolized in biomedical applications. In addition, ultraviolet radiation (UV) has damaging effects on bioactive 
species (Jun et al., 2014). It was demonstrated by Hwang et al. that the concentration of photoinitiator has adverse impacts on the cell viability (Hwang et al., 2008b). The negative aspects of UV-light can be minimized by decreasing the exposure time and using less-harmful wavelengths than the standard one (Panda et al., 2008). Due to these limitations, phtopolymerization is not the most desired approach for fabrication of fibers in cell encapsulation applications.

Although some thermoplastic polymers have been used in microfluidic fiber fabrication such as PLGA (Hwang et al., 2008a) and poly(methyl methacrylate) (PMMA) (Thangawng et al., 2009), there is no report on microfluidic fabrication of PCL fibers. PCL is a Food and Drug Administration (FDA) approved polymer which is widely used as a biomaterial due to its biocompatibility and biodegradability (Acar et al., 2014; Li et al., 2014; Soliman et al., 2010). Due to slower degradation rate of this polymer, for instance compared to PLGA, it possesses no adverse impacts on cell viability and migration because it does not change the $\mathrm{PH}$ of the environment during the degradation sharply (Sung et al., 2004). This polymer also has good mechanical properties, is not toxic, and its rate of degradation can be controlled. Furthermore, PCL does not trigger immune responses in the body (Hong and Kim, 2013).

In this paper, we have employed solvent extraction to fabricate biocompatible and biodegradable PCL microfibers in a microfluidic platform for the first time. PCL grants us the advantage of having a biocompatible and strong material from which to make fibers. Using microfluidics, we are able to avoid the constraints of other methods such as electrospinning (Hwang et al., 2008a). We can produce fibers with different cross-sectional shapes while the fabrication is continuous and stops only when the core and sheath solutions stop flowing. By fabricating PCL using a microfluidic microchannel, we are benefitting from combining the properties of a proven biocompatible material and the unique properties of a microfluidic fabrication technique to create fibers for many biomedical applications such as tissue engineering and drug delivery. 
After solidification of PCL fibers, they exit the channel directly into a water bath where the sheath fluid is washed off. The fibers are then collected and characterized to understand their physical and mechanical properties. We have also performed simulations using COMSOL multiphysics to compare with the experimental results.

\section{Materials}

Polycaprolactone $\left(\mathrm{M}_{\mathrm{n}}=80,000\right)$ and polyethylene glycol (PEG) $\left(\mathrm{M}_{\mathrm{n}}=20,000\right)$ were obtained from Sigma Aldrich (St. Louis, MO). The solvent for the core solution is 2,2,2- trifluoroethanol (TFE), which was purchased from Oakwood Chemical (West Columbia, SC). The material used for the microfluidic channel is polydimethylsiloxane (PDMS). The core solution was prepared by pouring PCL into the TFE at different concentrations by volume (2\%, 5\%, and $8 \%$ PCL). The sheath fluid was made using a PEG concentration of 5\% into a mixture of water and ethanol with a volume ratio of 1:1. The solutions were prepared at room temperature. The syringes used to pump the fluids were obtained from BD Medical (Franklin Lakes, New Jersey). The syringe pumps used to introduce fluids to the microchannel were purchased from Cole Parmer (Vernon Hills, Illinois).

\section{Microfluidic Approach}

Using a dual-drive syringe pump, the core and sheath fluids are simultaneously introduced into the microchannel. The core fluid enters the channel through the central opening and the sheath flow enters on each of the sides. Additionally, our microchannel contains three inlets, one in the middle for introducing the core flow and two on the sides for running sheath flows. (Figure 1). The shear force between the core and sheath fluids focuses the core fluid in the center of the microchannel hydrodynamically (Asrar et al., 2015; Hashemi et al., 2011a; Hashemi et al., 2011b; Hashemi et al., 2010) and fibers are formed from the core fluid employing solvent extraction solidification 
approach. The microfluidic method enables the fabrication of fibers with different sizes and cross sections. The dimensions of the fiber depend on the core and sheath flow rates (Zarrin and Dovichi, 1985). Additionally, the shear force aligns the polymer chains along the flow direction in the final microfiber product. Therefore, we can control the microstructures of the fibers and their bulk mechanical properties. Here, the phase inversion process causes the TFE in the core fluid to be replaced by the sheath fluid. Because PCL does not dissolve in the sheath fluid, it becomes solidified as a microfiber further downstream the channel.

Figure 1 (a) illustrates a schematic of microfluidic fiber fabrication. More details about the design of the microchannels are provided in Figures $\mathbf{S 1}$ and $\mathbf{S 2}$. The pattern of the core fluid at different sections of the channel is illustrated by Figure 1 (b). In fact, the sheath fluid has a lateral hydrodynamic force on the core fluid in the nozzle area. After that, the cross section of the channel remains constant until two fluids meet the chevrons of the channel. In this region, the hydrodynamic resistance in the direction parallel to the peaks and valleys is less than the direction of the channel. Consequently, the component of velocity which is perpendicular to the channel increases for both of the fluids. The hydrodynamic resistance is inversely proportional to the flow rate. Therefore, the sheath fluid will experience less resistance compared to the core fluid since the flow rate of the sheath fluid is considerably higher than the core fluid. Sheath fluid wraps around the core fluid and exerts vertical hydrodynamic force towards the center of the channel in the chevrons region. After passing through these chevrons, the core fluid will be focused at the center of the channel and there will not be any contact between the core fluid and the channel walls. Apart from exerting hydrodynamic force, the sheath fluid plays the role of a lubricant in the channel to facilitate fiber extrusion. Therefore, the viscosity of the sheath fluid should be matched to that of the core fluid. For this purpose, polyethylene glycol (PEG) is added to the sheath fluid in order to increase its viscosity. Figure 2 shows the viscosity of core and sheath fluids at different concentrations of PCL 
and PEG (2\%-8\%), respectively. While the range of viscosity varies from $19 \mathrm{cP}$ to $500 \mathrm{cP}$ for the core fluid, it is limited to $2-16 \mathrm{cP}$ for the sheath fluid. In this study, we used 5\% PEG in the sheath fluid and changed the concentration of the PCL in the core fluid from $2 \%$ to $8 \%$ in order to show the versatility of microfluidic approach in fabricating fiber using a wide range of core fluid viscosity.

While the core flow rate is kept constant at $5 \mu \mathrm{L} / \mathrm{min}$, the sheath flow rate varies from $10 \mu \mathrm{L} / \mathrm{min}$ to $120 \mu \mathrm{L} / \mathrm{min}$. Our microchannel contains three inlets, one in the middle for introducing the core flow and two on the sides for running sheath flows. The channel has four chevron grooves that create vertical hydrodynamic force. The magnitude of the hydrodynamic force is directly related to the flow rate ratio (velocity gradient between sheath and core fluid), which is an important parameter in determining the features of the fiber.

Downstream from the microchannel entrance, the fluid comes into contact with the chevron grooves, which are engrained on the top and bottom surfaces of the channel. The grooves play an important role in focusing the core fluid vertically and determining the final cross-sectional shape of the fibers. The shear stress aligns the polymer chains in the core flow in the direction of the flows. The solidification of core flow occurs once it comes to contact with the sheath flows. During the whole process, the channel is positioned vertically and the resulting fibers exit directly into a water bath.

After the fibers were recovered, their characteristics were evaluated through several means. A scanning electron microscope (SEM) from Nikon (Tokyo, Japan) was used to determine the morphology of the fibers. The SEM was used to study the effects of changing the flow rate ratio of sheath and core fluids as well as the PCL concentration in the core solution on the morphology and cross section of the fabricated microfibers. In addition, the mechanical properties of the fibers were 
measured to assess their strength. Finally, the microfluidic fiber fabrication was simulated using COMSOL multiphysics software in order to compare the experimental and numerical results.

\section{Characterization}

The morphology and cross section of the microfibers were studied using the field emission scanning electron microscopy (FE-SEM) (JSM-6700F at an acceleration voltage of $5 \mathrm{kV}$ ). The viscosities of the core and sheath solutions were measured using a digital viscometer (DV-E, Brookfield Engineering Laboratories, Inc., Middleboro, MA). For measuring the stress-strain behavior of the fibers, single fiber was tested using Instron Universal Testing machine (Model 5569, Instron Engineering Corp., Canton, MA). For each type of the fibers, 10 samples were tested and the average values for each type were reported. Since the PCL fibers have high ductility, we used a $10 \mathrm{~N}$ load cell to get enough resolution, and the extension rate was set to $20 \mathrm{~mm} / \mathrm{min}$. In this test, the samples were prepared by attaching them on a paper frame in order to be gripped properly by the Instron machine (Figure S3). After mounting the sample on the machine, we cut two sides of the frame to get the mechanical properties of PCL fiber. The length of the samples for this test was $15 \mathrm{~mm}$. The results were found using Bluehill software. A video file is provided in supplementary materials, that shows high ductility of the fiber during the tensile test. The stress-strain curves were fitted with linear line for the elastic region. Second order polynomial equation was used for the plastic region because its coefficient of determination (R-Square) was better compared to the linear line for all of the data.

\section{Results and Discussion}

We fabricated microfibers using 2\%, 5\%, and 8\% PCL and 5\% PEG in TFE and water/ethanol (with the volume ratio of 1:1), respectively. Varying the sheath and core flow rates, it was found that the 
core flow rate of $5 \mu \mathrm{L} / \mathrm{min}$ and the sheath flow rate of $10 \mu \mathrm{L} / \mathrm{min}$ to $120 \mu \mathrm{L} / \mathrm{min}$ are appropriate flow rates in order to obtain continuous microfiber.

The versatility of the microfluidic fiber fabrication method can be highlighted by using various flow rates of sheath fluid and PCL concentrations in the core fluid. The results revealed that the flow rate ratio of the core and sheath fluids plays a significant role in the morphology of the fabricated fibers. Using a very low sheath flow rate does not allow for enough of both the vertical and horizontal hydrodynamic forces on the core fluid. As a result, the width of the core fluid increases. Also, the aggregation of the polymer becomes stronger than its elongation along the channel. This condition leads to occurrence of clogging in the microchannel. On the other hand, if the core fluid has a high flow rate, there is a possibility that the core fluid exit the channel and no solidification happens. Figure 3 shows the SEM images of the representative microfibers obtained using different flow rate ratios. The concentrations of PCL and PEG were kept at a constant value of $5 \%$ in the core and sheath fluids, respectively.

This figure illustrates that the roughness of the surface is not significantly affected by changing the flow rate ratio between the fluids. However, at the higher flow rates of sheath fluid, the microfibers have wavy structures, and as the sheath flow rate decreases, the microfibers tend to be more uniform and straight. This means that the Kelvin-Helmholtz instability occurs at high flow rate ratios due to sharp difference of velocities at the core fluid / sheath fluid interface in the channel, and it leads to the creation of wavy shaped microfibers. Additionally, this figure demonstrates that the size of the microfibers increases when the sheath flow rate reduces. This is expected, because when the difference of the velocities decreases, the hydrodynamic shear force exerted from the sheath fluid on the core fluid weakens. Consequently, the core fluid expands in the channel and the average diameter of the fiber increases. 
In Figure 4 (a-c), the cross-sectional SEM images of the microfibers fabricated with different flow rate ratios are provided. This figure shows that the cross-section of the fibers made by microfluidic approach can be tuned by simply changing the flow rate ratio between the sheath and core fluids. Additionally, the dimensions of the fibers (average \pm standard error) are shown in Figure 4 (d). The width and height of the PCL fiber at the sheath-to-core flow rate ratio of 120:5 are $16.21 \mu \mathrm{m}$ and $22.34 \mu \mathrm{m}$, respectively. The decrease of the flow rate ratio to $10: 5$ increases both the width and height of the fiber to $21.12 \mu \mathrm{m}$ and $33.56 \mu \mathrm{m}$, respectively. Moreover, the aspect ratio of the fiber increases by a factor of 1.15 when the flow rate ratio reduces from $120: 5$ to $10: 5$. That was expected because when the flow rate ratio between two fluids diminishes, the lateral hydrodynamic force exerted to the core fluid by sheath fluid weakens. Consequently, the core fluid has more freedom to grow in the lateral direction. On the other hand, when the sheath flow rate increases, the shear force intensifies and the core fluid is stretched more due to the higher hydrodynamic force that leads to the fabrication of fibers with smaller size.

The microfluidic fiber fabrication was simulated using COMSOL multiphysics. The NavierStokes equation for incompressible flow at steady state was used to numerically solve the momentum balance. Because the inertial forces are negligible at low Reynolds number, the motion of the fluid can be approximately described by the reversible Stokes equation in which the nonlinear term can be neglected. We used Fick's law, $-\nabla \cdot(-D . \nabla c)+u \cdot \nabla c=0$, to describe the diffusive transport in the micro-channel. In this equation, $\mathrm{D}$ is the diffusion coefficient and $\mathrm{c}$ represents the concentration. The Navier-Stokes equation was solved first and was then followed by the convection-diffusion relationships. Due to symmetry, one fourth of the channel was modeled. The velocity in different sections of the channel is provided in Figure S2. This figure illustrates that the velocity of two fluids increases by passing through the nozzle part of the channel. In the chevrons area, the component of velocity which is perpendicular to the channel, increases by passing the fluid 
through the chevrons. Figure 5 (a) shows the concentration distribution along the channel. The bright and dark colors represent the situations of core and sheath fluids, respectively along the channel. Therefore, the effects of lateral and vertical hydrodynamic focusing forces of the sheath fluid on the core fluid can be observed clearly by following the brighter color along the channel. Additionally, the bright color at the output of the microchannel represents the cross sectional pattern of the microfiber fabricated using a specific flow rate ratio. Based on Figure 5 (a), the thickness of the core fluid reduces after the nozzle area, which reveals an increasing lateral force of the sheath fluid on the core fluid. Downstream from the initial focusing region, the series of chevrons change the hydrodynamic resistance in the channel such that the resistance in the perpendicular direction becomes smaller than the parallel one. Figure 5 (a) displays that the vertical force in the chevrons region gradually focuses the core solution at the center of the channel.

The concentration distribution of the core and sheath fluids are shown in Figure $\mathbf{5}\left(b_{1}-d_{1}\right)$ for different values of sheath flow-rate and constant value of $5 \mu \mathrm{L} / \mathrm{min}$ for the core flow rate. This figure illustrates a weakening of the hydrodynamic lateral force of the sheath fluid on the core fluid due to decreasing the sheath flow-rate. Consequently, the width of the fabricated fiber becomes larger. The vertical hydrodynamic force, however, does not change significantly due to the fact that this force is mostly originated from the depth of the chevrons. Therefore, the combination of a decrease in lateral force and a constant value of the vertical force leads to the development of the ribbon-shape pattern. Figure $5\left(b_{2}-d_{2}\right)$ illustrates the trend in which the core cross section changes to a ribbon-shaped pattern. These results demonstrated consistency between the experimental and numerical results.

The concentration of the core fluid can be changed in microfluidic fiber fabrication as well as the flow rate and flow rate ratio between the sheath and core fluid in order to change the characteristics of microfibers. Figure 6 illustrates the effects of different concentrations $(2 \%, 5 \%$, 
and $8 \%$ ) of PCL in the core fluid on morphology of fibers. Increasing the PCL concentration results in fiber with smoother surface. Additionally, 2\% PCL fibers show more porosity compared to the fibers made from higher PCL concentrations. When core fluid with low concentration of PCL is introduced into the channel, the total amount of PCL in core fluid is not enough to create a uniform fiber after solvent extraction and the resulting fibers become more porous. Moreover, the higher roughness and existence of porosity on the fiber at low concentrations of PCL is due to rapid exchange of TFE and sheath fluid compared to higher concentrations. While, the uniform microfibers have higher mechanical properties, more porous microfibers can enhance cell adhesion and cell proliferation, which are desirable in tissue engineering applications. Furthermore, tuning the porosity and microstructures of the fibers by changing the PCL concentration in the core fluid is another advantage of microfluidic approach. The cross-sectional SEM images of PCL microfibers fabricated using different percentages of PCL in the core fluid are shown in Figure 7 (a-c). This figure demonstrates that the PCL concentration can influence the size of the resulting fiber as well as the flow rate ratio between the two fluids. Figure 7 (d) displays the dimensions of the fibers made by different PCL concentration in the core fluid. We observed that the dimension of the fiber (width $\times$ height) increases from $20.1 \mu \mathrm{m} \times 13.3 \mu \mathrm{m}$ to $33.65 \mu \mathrm{m} \times 24.25 \mu \mathrm{m}$ when the PCL concentration changes from $2 \%$ to $8 \%$, while the aspect ratio does not change significantly. That is because when lower amount of PCL in the core solution flows through the channel, the dimensions of PCL solidified as a fiber during the phase inversion process in the microchannel will be reduced.

The mechanical properties of the fibers made by different flow rate ratios were investigated. Figure S3 illustrates a single fiber prepared for the tensile test. Stress-strain behavior of different fibers are shown in Figure 8 (a). This figure demonstrates a wide range of mechanical properties that can be obtained using this microfluidic approach. As expected from a typical plastic material, the elastic region of the PCL stress-strain curve is in a small range, which is shown in Figure 8 (b). 
The Young's modulus of the fibers are shown in Figure 8 (c). Additionally, yield strain (\%), yield stress $(\mathrm{MPa})$, Young's modulus $(\mathrm{MPa})$, strain at break $(\%)$, and stress at break $(\mathrm{MPa})$ are listed in Table 1 for different PCL microfibers. The results show that the decrease of the flow rate ratio from 40:5 to 10:5, significantly improves the tensile stress at break, tensile strain at break, and the Young's modulus from 24.51 $\mathrm{MPa}$ to $77.07 \mathrm{MPa}, 567 \%$ to $1420 \%$, and $247.25 \mathrm{MPa}$ to $539.70 \mathrm{MPa}$, respectively. The yield stress (MPa) and yield elongation (\%) improve by a factor of 3.33 and 1.45 when the flow rate ratio decreases from 40:5 to 20:5.

Although the mechanical properties of electrospun PCL fibrous scaffold widely have been studied, there are few reports about the mechanical properties of PCL single fibers made by electrospinning method. It was found that the reported values of the tensile strain at break (\%)for electrospun fibers are significantly lower than our results(Baker et al., 2016; Croisier et al., 2012; Duling et al., 2008; Ghasemi-Mobarakeh et al., 2008; Sing Yian et al., 2006). This could be due to the microstructure organization of the fibers as the shear stress plays a pivotal role in aligning the polymer chains in the direction of the flow and consequently creating highly structured fibers. However, the values of stress at break $(\mathrm{MPa})$ and Young's modulus obtained in this study are comparable with the ones reported for the electrospun PCL fibers. Also based on the SEM images of microfiber cross sections shown in Figure 4, decreasing the flow rate ratio leads to an increase in the size and aspect ratio of the fiber cross section such that the fiber cross section tends to have a ribbon shape. Consequently, this improvement in mechanical properties of microfibers can be due to the ribbon shape of the microfiber cross section.

\section{Conclusions}

PCL microfibers with improved mechanical properties were fabricated using the microfluidic fiber fabrication. Employing microfluidic fiber fabrication approach, we created microfibers with the 
maximum strain of $1420 \%$. We showed through SEM that the morphology and size of the fibers could be controlled by varying the PCL percentage in the core solution and the flow rate ratio of sheath to core fluids. While the smoothness of the fiber was improved by increasing the PCL concentration in the core solution from $2 \%$ to $8 \%$, the flow rate ratio did not have a substantial influence on the roughness of the fiber. The aspect ratio of the fiber increases by diminishing the flow rate ratio because when the sheath flow rate decreases, the core fluid expands in the channel, which increases the width of the fiber. Numerical simulations were consistent with the experimental results. This development in size and cross section of the fiber enhanced the mechanical properties of the microfiber. These are the most improved properties compared to those of the previous reports about PCL fibers created using other fabrication methods. This improvement reveals unique capability of microfluidic platform to create fibers with a wide range of mechanical properties simply by changing the fabrication parameters such as flow rate ratio and viscosity.

\section{Acknowledgements}

This work was funded in part by the Office of Naval Research Grant N000141612246, Iowa State University Presidential Initiative for Interdisciplinary Research, and U.S. Department of Energy

Office of Science under the Science Undergraduate Laboratory Internship (SULI) Program at Ames Laboratory. The authors also would like to take the opportunity to express their appreciation to Jeremy Caplin, Rui Ding, and Michelle Grawe for their assistance in this research.

\section{References}

Acar, H., Çınar, S., Thunga, M., Kessler, M.R., Hashemi, N., Montazami, R., 2014. Study of Physically Transient Insulating Materials as a Potential Platform for Transient Electronics and Bioelectronics. Advanced Functional Materials 24, 4135-4143.

Akbari, M., Sinton, D., Bahrami, M., 2011. Viscous flow in variable cross-section microchannels of arbitrary shapes. International Journal of Heat and Mass Transfer 54, 3970-3978. 
Asrar, P., Sucur, M., Hashemi, N., 2015. Multi-Pixel Photon Counters for Optofluidic Characterization of Particles and Microalgae. Biosensors 5, 308-318.

Bai, Z., Reyes, J.M.M., Montazami, R., Hashemi, N., 2014. On-chip development of hydrogel microfibers from round to square/ribbon shape. Journal of Materials Chemistry A 2, 4878-4884.

Baker, S.R., Banerjee, S., Bonin, K., Guthold, M., 2016. Determining the mechanical properties of electrospun poly- $\varepsilon$-caprolactone (PCL) nanofibers using AFM and a novel fiber anchoring technique. Materials Science and Engineering: C 59, 203-212.

Boyd, D.A., Shields, A.R., Howell, P.B., Ligler, F.S., 2013a. Design and fabrication of uniquely shaped thiol-ene microfibers using a two-stage hydrodynamic focusing design. Lab on a Chip 13, 3105-3110.

Boyd, D.A., Shields, A.R., Naciri, J., Ligler, F.S., 2013b. Hydrodynamic Shaping, Polymerization, and Subsequent Modification of Thiol Click Fibers. ACS Applied Materials \& Interfaces 5, 114-119.

Caplin, J.D., Granados, N.G., James, M.R., Montazami, R., Hashemi, N., 2015. Microfluidic Organ-on-aChip Technology for Advancement of Drug Development and Toxicology. Advanced Healthcare Materials 4, 1426-1450.

Cho, S., Shim, T.S., Yang, S.M., 2012. High-throughput optofluidic platforms for mosaicked microfibers toward multiplex analysis of biomolecules. Lab on a Chip 12, 3676-3679.

Choi, C.H., Yi, H., Hwang, S., Weitz, D.A., Lee, C.S., 2011. Microfluidic fabrication of complex-shaped microfibers by liquid template-aided multiphase microflow. Lab on a Chip 11, 1477-1483.

Chung, B.G., Lee, K.H., Khademhosseini, A., Lee, S.H., 2012. Microfluidic fabrication of microengineered hydrogels and their application in tissue engineering. Lab on a Chip 12, 45-59.

Croisier, F., Duwez, A.S., Jerome, C., Leonard, A.F., van der Werf, K.O., Dijkstra, P.J., Bennink, M.L., 2012. Mechanical testing of electrospun PCL fibers. Acta Biomaterialia 8, 218-224.

Daniele, M.A., Adams, A.A., Naciri, J., North, S.H., Ligler, F.S., 2014a. Interpenetrating networks based on gelatin methacrylamide and PEG formed using concurrent thiol click chemistries for hydrogel tissue engineering scaffolds. Biomaterials 35, 1845-1856.

Daniele, M.A., Boyd, D.A., Adams, A.A., Ligler, F.S., 2015. Microfluidic Strategies for Design and Assembly of Microfibers and Nanofibers with Tissue Engineering and Regenerative Medicine Applications. Advanced Healthcare Materials 4, 11-28.

Daniele, M.A., North, S.H., Naciri, J., Howell, P.B., Foulger, S.H., Ligler, F.S., Adams, A.A., 2013. Rapid and Continuous Hydrodynamically Controlled Fabrication of Biohybrid Microfibers. Advanced Functional Materials 23, 698-704.

Daniele, M.A., Radom, K., Ligler, F.S., Adams, A.A., 2014b. Microfluidic fabrication of multiaxial microvessels via hydrodynamic shaping. RSC Advances 4, 23440-23446.

Deng, M., James, R., Laurencin, C.T., Kumbar, S.G., 2012. Nanostructured Polymeric Scaffolds for Orthopaedic Regenerative Engineering. IEEE Trans. Nanobiosci. 11, 3-14.

Duling, R.R., Dupaix, R.B., Katsube, N., Lannutti, J., 2008. Mechanical characterization of electrospun polycaprolactone (PCL): A potential scaffold for tissue engineering. Journal of Biomechanical EngineeringTransactions of the Asme 130, 13.

Ella, V., Annala, T., Lansman, S., Nurminen, M., Kellomaki, M., 2011. Knitted polylactide 96/4 L/D structures and scaffolds for tissue engineering: shelf life, in vitro and in vivo studies. Biomatter 1, 102-113. Enea, D., Henson, F., Kew, S., Wardale, J., Getgood, A., Brooks, R., Rushton, N., 2011. Extruded collagen fibres for tissue engineering applications: effect of crosslinking method on mechanical and biological properties. Journal of Materials Science-Materials in Medicine 22, 1569-1578.

Ghasemi-Mobarakeh, L., Prabhakaran, M.P., Morshed, M., Nasr-Esfahani, M.-H., Ramakrishna, S., 2008.

Electrospun poly(epsilon-caprolactone)/gelatin nanofibrous scaffolds for nerve tissue engineering.

Biomaterials 29, 4532-4539.

Goodrich, P.J., Sharifi, F., Hashemi, N., 2015. Rapid prototyping of microchannels with surface patterns for fabrication of polymer fibers. RSC Advances 5, 71203-71209.

Hasani-Sadrabadi, M.M., VanDersarl, J.J., Dashtimoghadam, E., Bahlakeh, G., Majedi, F.S., Mokarram, N., Bertsch, A., Jacob, K.I., Renaud, P., 2013. A microfluidic approach to synthesizing high-performance microfibers with tunable anhydrous proton conductivity. Lab on a Chip 13, 4549-4553. 
Hashemi, N., Erickson, J.S., Golden, J.P., Jackson, K.M., Ligler, F.S., 2011a. Microflow Cytometer for optical analysis of phytoplankton. Biosensors and Bioelectronics 26, 4263-4269.

Hashemi, N., Erickson, J.S., Golden, J.P., Ligler, F.S., 2011b. Optofluidic characterization of marine algae using a microflow cytometer. Biomicrofluidics 5, 032009.

Hashemi, N., Howell, J.P.B., Erickson, J.S., Golden, J.P., Ligler, F.S., 2010. Dynamic reversibility of hydrodynamic focusing for recycling sheath fluid. Lab on a Chip 10, 1952-1959.

Hong, S.G., Kim, G.H., 2013. Mechanically improved electrospun PCL biocomposites reinforced with a collagen coating process: preparation, physical properties, and cellular activity. Bioprocess. Biosyst. Eng. 36, 205-214.

Hwang, C.M., Khademhosseini, A., Park, Y., Sun, K., Lee, S.-H., 2008a. Microfluidic Chip-Based Fabrication of PLGA Microfiber Scaffolds for Tissue Engineering. Langmuir 24, 6845-6851.

Hwang, D.K., Dendukuri, D., Doyle, P.S., 2008b. Microfluidic-based synthesis of non-spherical magnetic hydrogel microparticles. Lab on a Chip 8, 1640-1647.

Jeong, W., Kim, J., Kim, S., Lee, S., Mensing, G., Beebe, D.J., 2004. Hydrodynamic microfabrication via "on the fly" photopolymerization of microscale fibers and tubes. Lab on a Chip 4, 576-580.

Jun, Y., Kang, E., Chae, S., Lee, S.H., 2014. Microfluidic spinning of micro- and nano-scale fibers for tissue engineering. Lab on a Chip 14, 2145-2160.

Jung, J.-H., Choi, C.-H., Chung, S., Chung, Y.-M., Lee, C.-S., 2009. Microfluidic synthesis of a cell adhesive Janus polyurethane microfiber. Lab Chip 9, 2596-2602.

Kang, E., Jeong, G.S., Choi, Y.Y., Lee, K.H., Khademhosseini, A., Lee, S.H., 2011. Digitally tunable physicochemical coding of material composition and topography in continuous microfibres. Nature Materials 10, 877-883.

Khademhosseini, A., Langer, R., Borenstein, J., Vacanti, J.P., 2006. Microscale technologies for tissue engineering and biology. Proceedings of the National Academy of Sciences of the United States of America $103,2480-2487$.

Kraitzer, A., Ofek, L., Schreiber, R., Zilberman, M., 2008. Long-term in vitro study of paclitaxel-eluting bioresorbable core/shell fiber structures. Journal of Controlled Release 126, 139-148.

Li, Y.-F., Rubert, M., Aslan, H., Yu, Y., Howard, K.A., Dong, M., Besenbacher, F., Chen, M., 2014.

Ultraporous interweaving electrospun microfibers from PCL-PEO binary blends and their inflammatory responses. Nanoscale 6, 3392-3402.

Oh, H.J., Kim, S.H., Baek, J.Y., Seong, G.H., Lee, S.H., 2006. Hydrodynamic micro-encapsulation of aqueous fluids and cells via 'on the fly' photopolymerization. Journal of Micromechanics and Microengineering 16, 285-291.

Panda, P., Ali, S., Lo, E., Chung, B.G., Hatton, T.A., Khademhosseini, A., Doyle, P.S., 2008. Stop-flow lithography to generate cell-laden microgel particles. Lab on a Chip 8, 1056-1061.

Reddy, N., Yang, Y.Q., 2010. Structure and properties of cocoons and silk fibers produced by Hyalophora cecropia. Journal of Materials Science 45, 4414-4421.

Saraf, A., Baggett, L.S., Raphael, R.M., Kasper, F.K., Mikos, A.G., 2010. Regulated non-viral gene delivery from coaxial electrospun fiber mesh scaffolds. Journal of Controlled Release 143, 95-103.

Shin, S., Park, J.Y., Lee, J.Y., Park, H., Park, Y.D., Lee, K.B., Whang, C.M., Lee, S.H., 2007. "On the fly" continuous generation of alginate fibers using a microfluidic device. Langmuir 23, 9104-9108.

Sing Yian, C., Todd, C.H., Chwee Teck, L., Kam, W.L., 2006. Mechanical properties of single electrospun drug-encapsulated nanofibres. Nanotechnology 17, 3880.

Soliman, S., Pagliari, S., Rinaldi, A., Forte, G., Fiaccavento, R., Pagliari, F., Franzese, O., Minieri, M., Di Nardo, P., Licoccia, S., Traversa, E., 2010. Multiscale three-dimensional scaffolds for soft tissue engineering via multimodal electrospinning. Acta Biomaterialia 6, 1227-1237.

Sung, H.J., Meredith, C., Johnson, C., Galis, Z.S., 2004. The effect of scaffold degradation rate on threedimensional cell growth and angiogenesis. Biomaterials 25, 5735-5742.

Tamayol, A., Akbari, M., Annabi, N., Paul, A., Khademhosseini, A., Juncker, D., 2013. Fiber-based tissue engineering: Progress, challenges, and opportunities. Biotechnol. Adv. 31, 669-687. 
Thangawng, A.L., Howell, P.B., Richards, J.J., Erickson, J.S., Ligler, F.S., 2009. A simple sheath-flow microfluidic device for micro/nanomanufacturing: fabrication of hydrodynamically shaped polymer fibers. Lab on a Chip 9, 3126-3130.

Tiwari, S.K., Tzezana, R., Zussman, E., Venkatraman, S.S., 2010. Optimizing partition-controlled drug release from electrospun core-shell fibers. International Journal of Pharmaceutics 392, 209-217.

Vunjak-Novakovic, G., Altman, G., Horan, R., Kaplan, D.L., 2004. Tissue engineering of ligaments. Annual Review of Biomedical Engineering 6, 131-156.

Yim, E.K.F., Wan, A.C.A., Le Visage, C., Liao, I.C., Leong, K.W., 2006. Proliferation and differentiation of human mesenchymal stem cell encapsulated in polyelectrolyte complexation fibrous scaffold. Biomaterials 27, 6111-6122.

Yoo, I., Song, S., Uh, K., Lee, C.W., Kim, J.-M., 2015. Size-Controlled Fabrication of Polyaniline Microfibers Based on 3D Hydrodynamic Focusing Approach. Macromolecular Rapid Communications 36, 1272-1276.

Yoo, I., Song, S., Yoon, B., Kim, J.-M., 2012. Size-Controlled Fabrication of Polydiacetylene-Embedded Microfibers on a Microfluidic Chip. Macromolecular Rapid Communications 33, 1256-1261.

Zarrin, F., Dovichi, N.J., 1985. Sub-picoliter detection with the sheath flow cuvette. Analytical Chemistry 57, 2690-2692. 

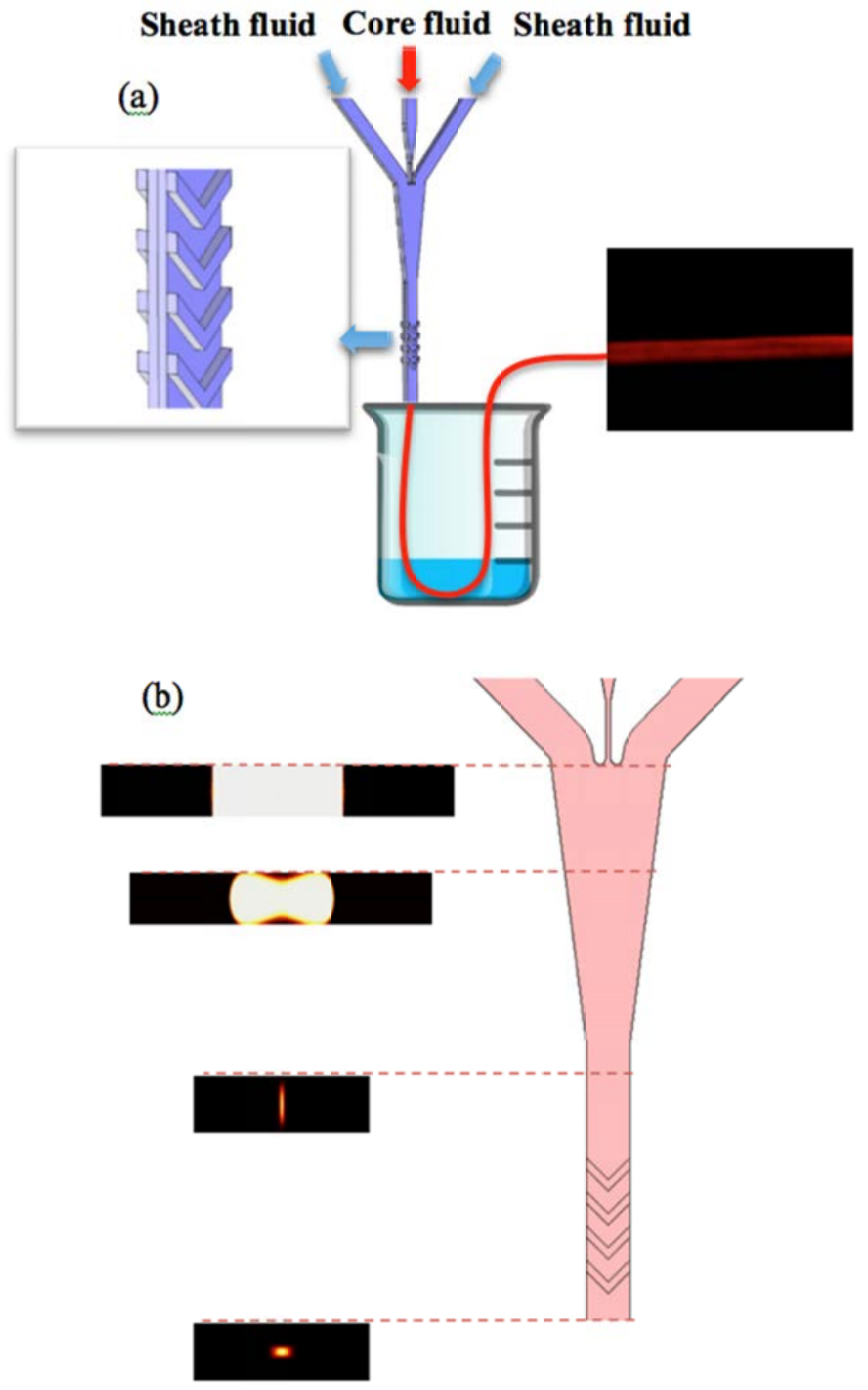

Figure 1. (a) A schematic of microfluidic fiber fabrication method. (b) Concentration pattern of the core fluid at different sections of the channel; the flow rate ratio of $80: 5 \mu \mathrm{L} / \mathrm{min}$ for the sheath and core fluids, respectively. 


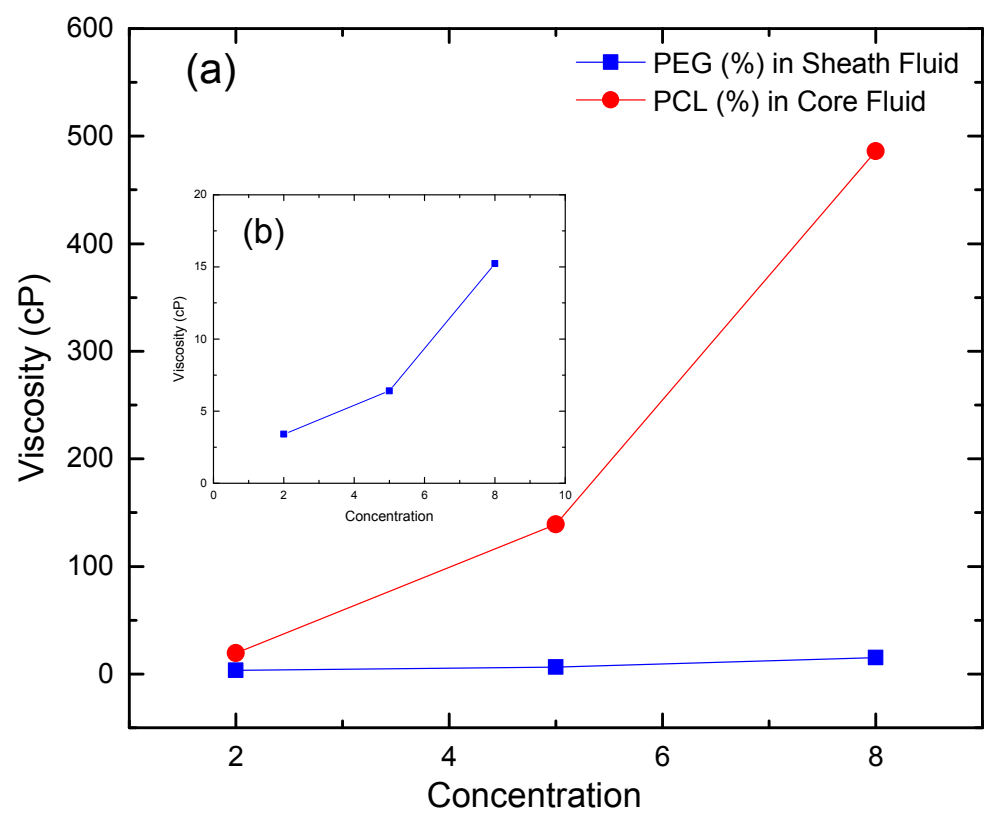

Figure 2. Viscosity of (a) the core and sheath solutions using different concentrations of PCL and PEG, respectively; (b) enlarged view of sheath solution viscosity made by three different concentrations of PEG. 

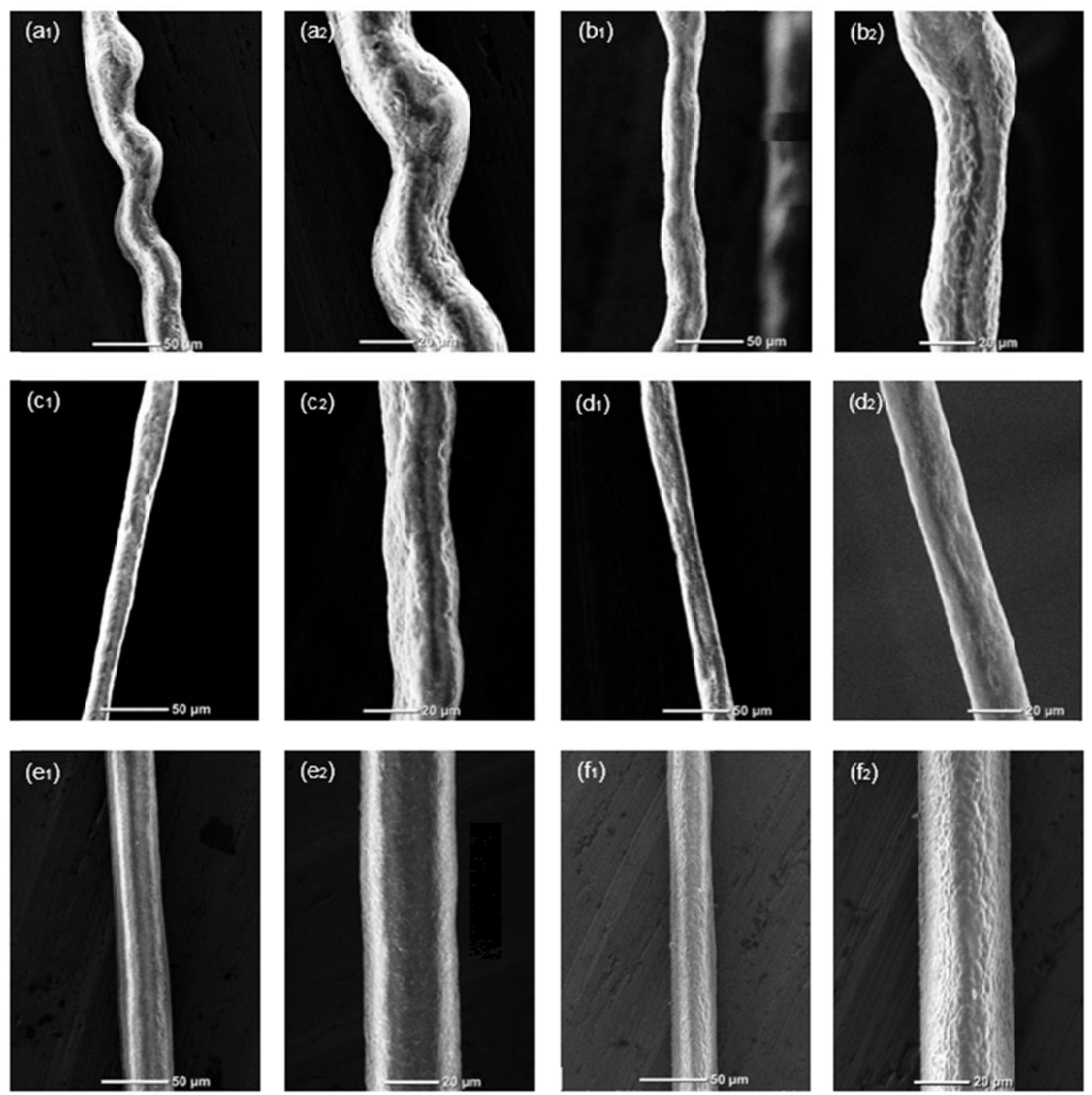

Figure 3. SEM images of PCL microfibers with 5\% PCL in TFE (core fluid) and 5\% PEG in water and ethanol (sheath fluid) and different flow rates of (a) 120: 5 (b) 100: 5 (c) $80: 5$ (d) 60: 5 (e) 20: 5, and (f) 10: $5 \mu \mathrm{L} / \mathrm{min}$ for the sheath and core fluids, respectively. 

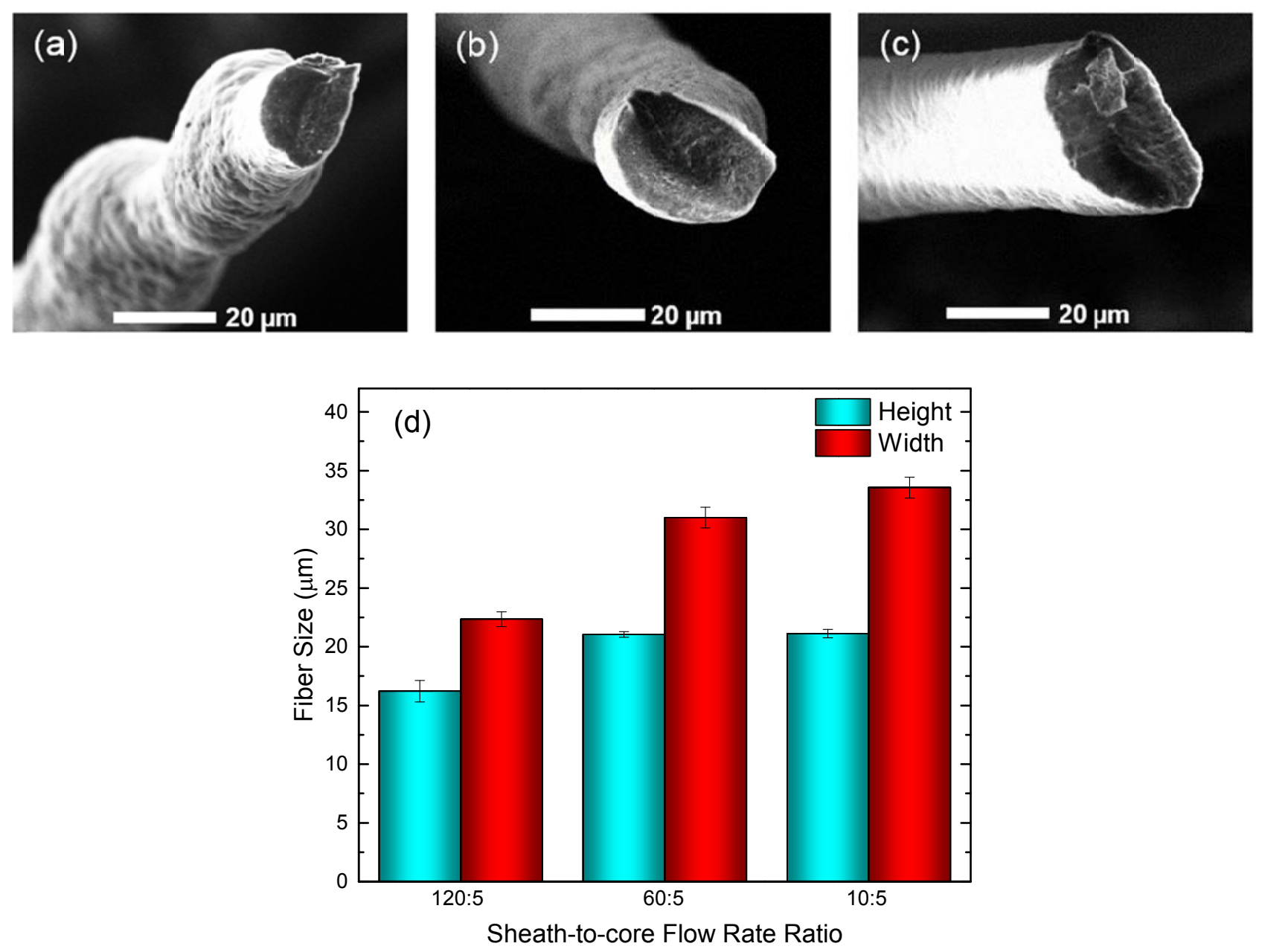

Figure 4. Cross sectional SEM images of PCL microfibers with 5\% PCL in TFE (core fluid) and 5\% PEG in water and ethanol (sheath fluid) fabricated by sheath and core flow rates of (a) 120:5, (b) 60:5, and (c) 10:5 $\mu \mathrm{L} / \mathrm{min}$, respectively. (d) Dimensions of the PCL fibers fabricated using different sheath-to-core flow rate ratios. 

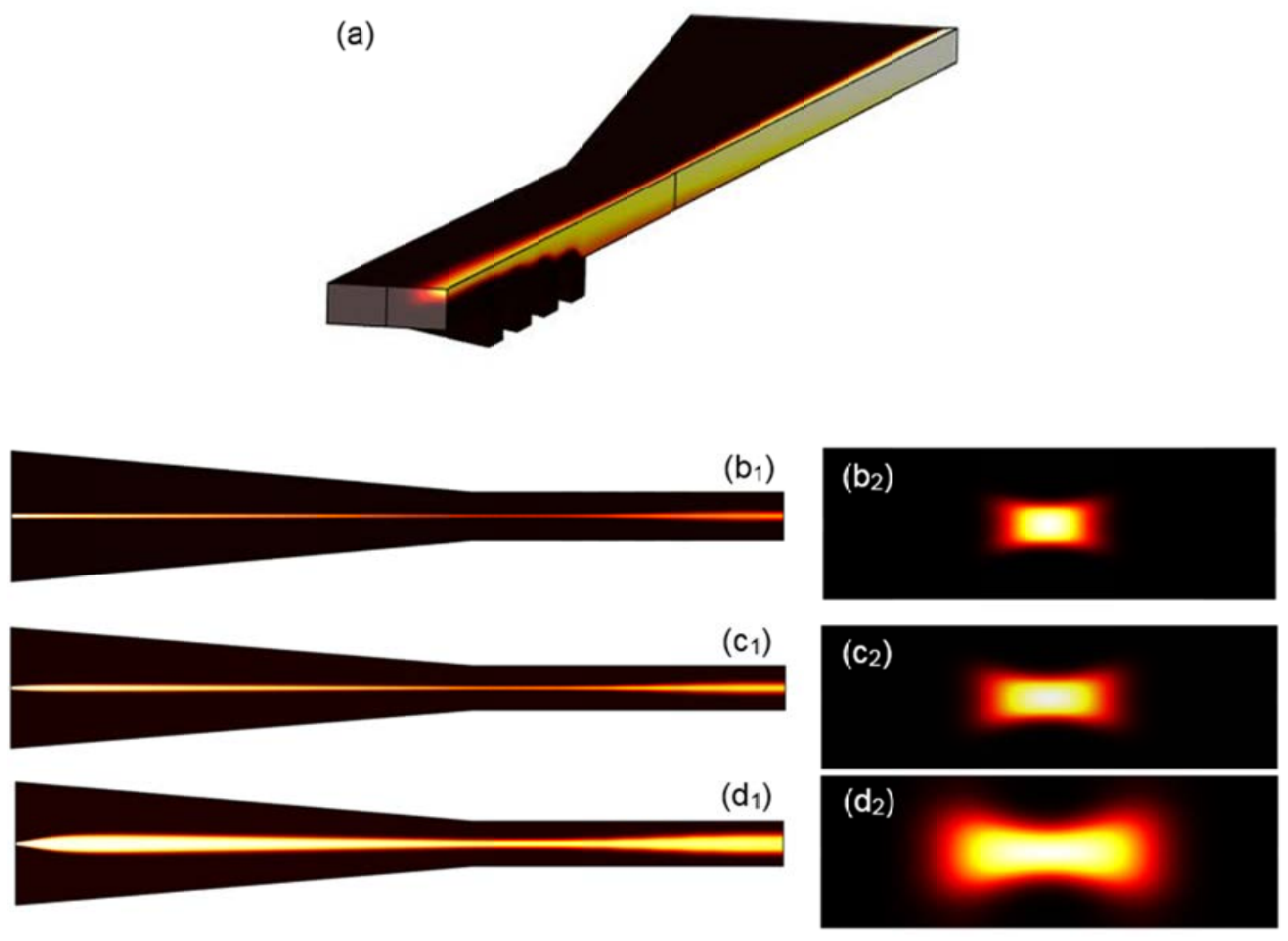

Figure 5. (a) Three-dimensional concentration distribution of core fluid in sheath fluid along the channel; the flow rate ratio is $80: 5 \mu \mathrm{L} / \mathrm{min}$ for the sheath and core fluids, respectively; Top view of the channel and the cross section of the fibers with sheath and core flow-rates of $\left(b_{1}\right.$ and $\left.b_{2}\right) 120: 5$, $\left(c_{1}\right.$ and $\left.c_{2}\right) 60: 5$, and $\left(d_{1}\right.$ and $\left.d_{2}\right) 10: 5 \mu \mathrm{L} / \mathrm{min}$, respectively. 

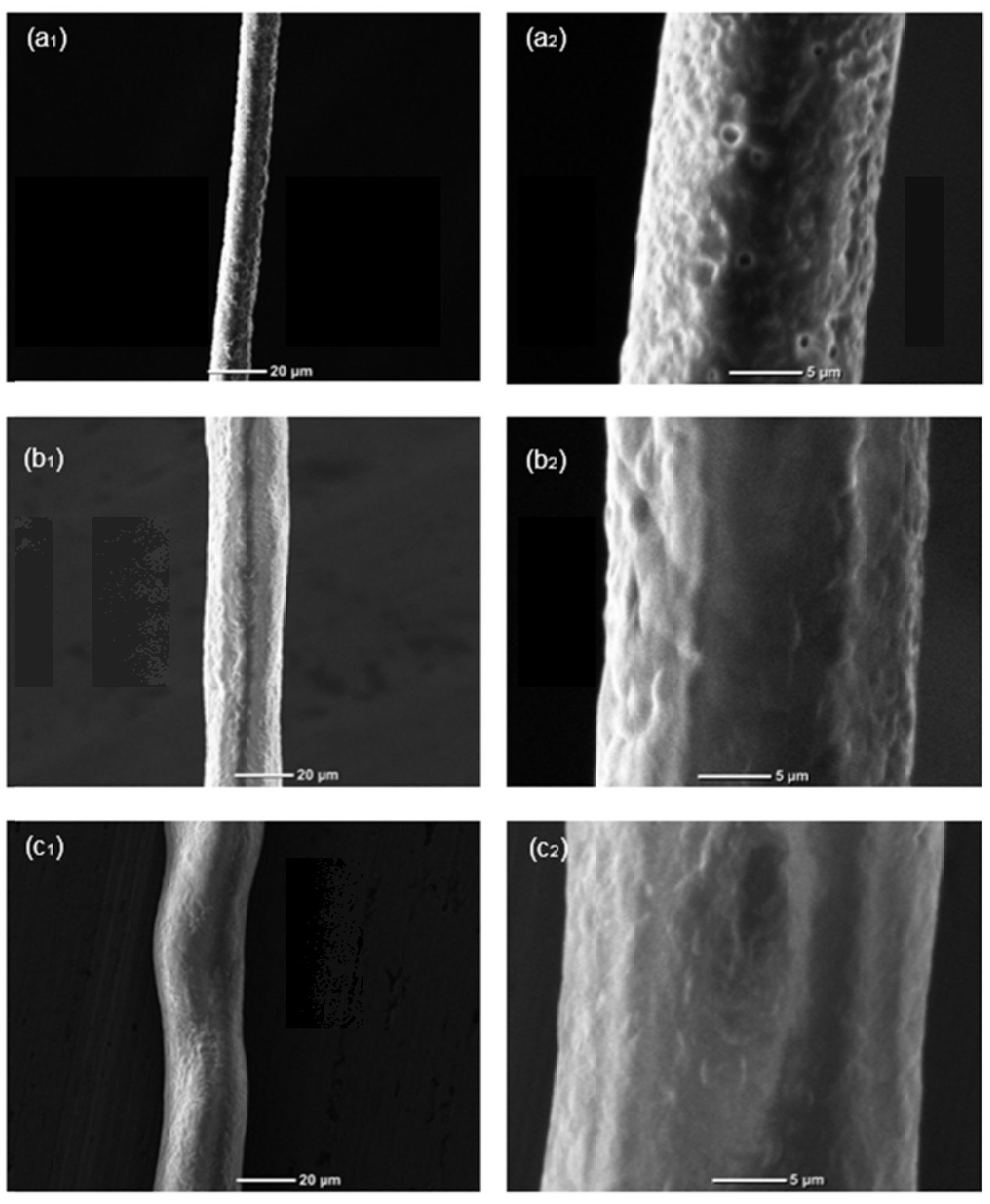

Figure 6. SEM images of PCL microfibers with 5\% PEG in the sheath fluid and the PCL concentrations of $\left(a_{1}-a_{2}\right) 2 \% ;\left(b_{1}-b_{2}\right) 5 \%$; and $\left(c_{1}-c_{2}\right) 8 \%$ in the core fluid. Sheath flow rate is 60 $\mu \mathrm{L} / \mathrm{min}$ and core flow rate id $5 \mu \mathrm{L} / \mathrm{min}$. 

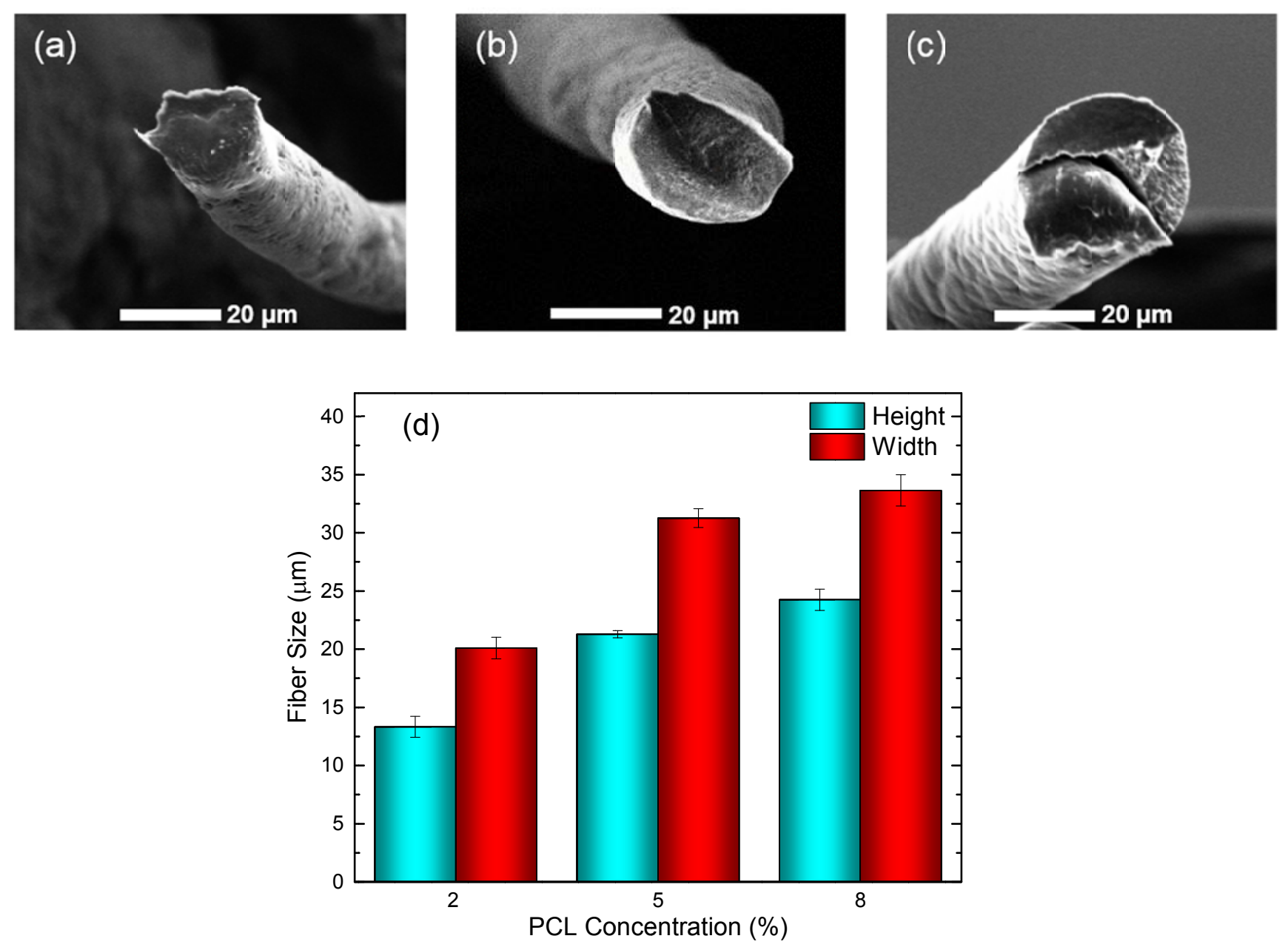

Figure 7. Cross sectional SEM images of PCL microfibers with different concentrations of (a) $2 \%$; (b) 5\%; and (c) $8 \%$ PCL in the core fluid and 5\% PEG in the sheath fluid with the flow rate of 60:5 $\mu \mathrm{L} / \mathrm{min}$ for the sheath and core fluids, respectively. (d) Dimensions of the PCL fibers fabricated using different PCL concentrations in the core fluid. 

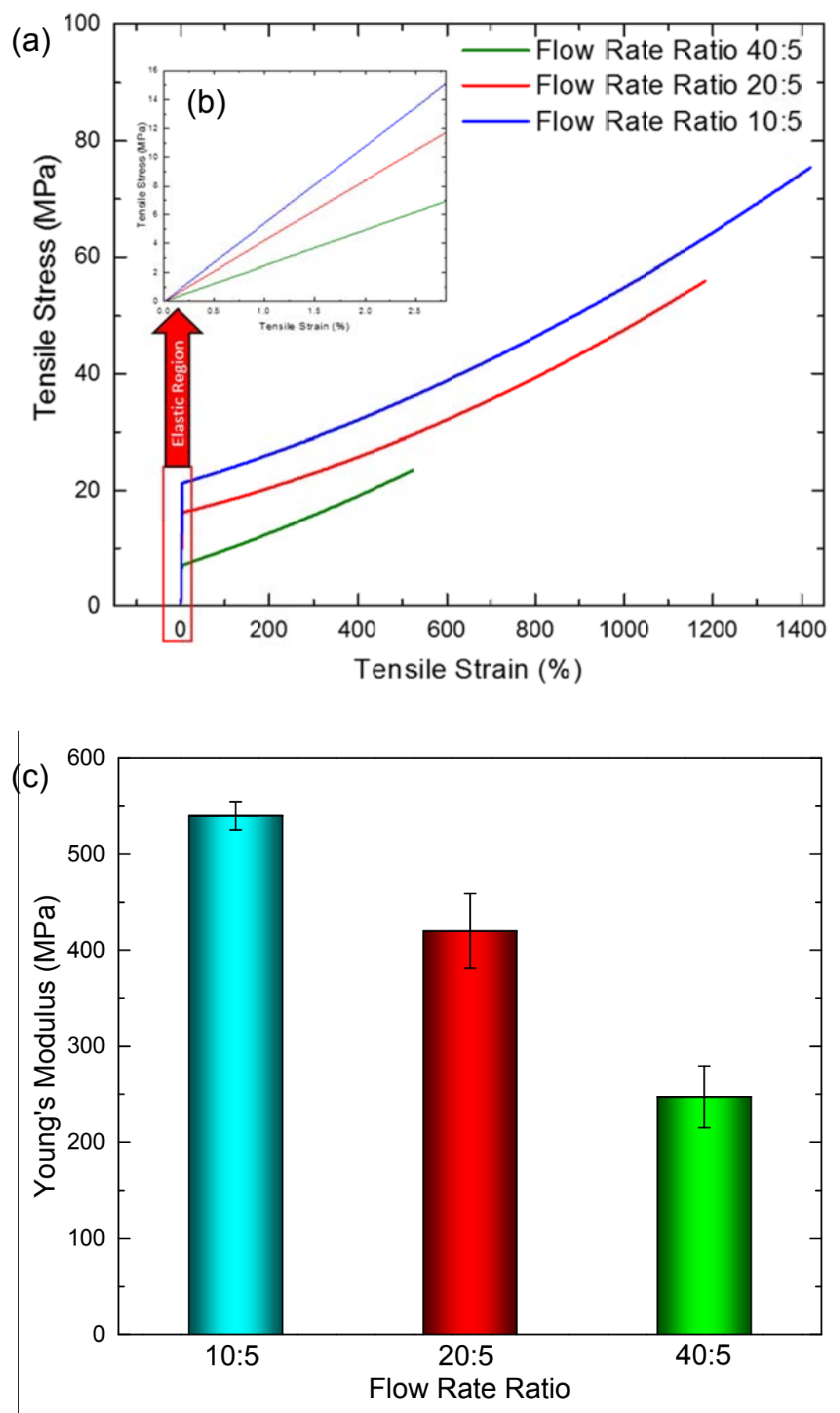

Figure 8. (a) Tensile stress - strain behavior, (b) enlarged view of the elastic region of stress-strain curve, and(c) Young's modulus of PCL microfibers fabricated with different flow rate ratios with the PCL concentration of $5 \%$ in TFE. 
Table 1 Mechanical properties of PCL fibers made by different sheath-to-core flow rate ratios

\begin{tabular}{llllll}
$\begin{array}{l}\text { Flow Rate } \\
\text { Ratio }\end{array}$ & $\begin{array}{l}\text { Yield Strain } \\
(\%)\end{array}$ & $\begin{array}{l}\text { Yield Stress } \\
\text { (MPa) }\end{array}$ & $\begin{array}{l}\text { Young's } \\
\text { Modulus }\end{array}$ & $\begin{array}{l}\text { Strain at Break } \\
(\%)\end{array}$ & $\begin{array}{l}\text { Stress at } \\
\text { Break (MPa) }\end{array}$ \\
\hline $\mathbf{4 0 : 5}$ & $2.70 \pm 0.65$ & $6.02 \pm 1.02$ & $247.25 \pm 32.08$ & $567 \pm 61.24$ & $24.51 \pm 3.11$ \\
\hline $\mathbf{2 0 : 5}$ & $3.78 \pm 0.24$ & $15.78 \pm 1.50$ & $420.03 \pm 38.89$ & $1079.25 \pm 63.20$ & $57.35 \pm 5.46$ \\
\hline $\mathbf{1 0 : 5}$ & $3.92 \pm 0.33$ & $20.05 \pm 1.15$ & $539.70 \pm 14.50$ & $1420.4 \pm 79.47$ & $77.07 \pm 5.64$ \\
\hline
\end{tabular}

